\title{
A GENERIC APPROACH FOR A MICRO PARTS FEEDING SYSTEM
}

\author{
M. Paris ${ }^{1}$, C. Perrard ${ }^{1}$, P. Lutz ${ }^{1}$ \\ ${ }^{1}$ Laboratoire d'Automatique de Besançon - UMR CNRS 6596 - ENSMM - UFC \\ 24 rue Alain Savary, 25000 Besançon, France
}

\begin{abstract}
The paper propose a new approach in order to design a generic microparts feeder. The method based on a classification scheme allows to emerge the principal characteristics of each studies solutions. The different criteria take into account the specifities of the micro world and moreover the main characteristics for the feeding functions. Thus, we analyse three systems and confront them to find the generic and flexibility aspects.
\end{abstract}

Keywords: Feeding, classification scheme, microfactory.

\section{INTRODUCTION}

It is within the framework of European project EUPASS (Evolvable Ultra-Precision Assembly SystemS) that the whole of work presented here were carried out. The main objectives are to realize a new generation of modular high-precision manipulators, grippers and feeders which will work inside the microworld. As in any system of production, it is essential to take into account the constraints of production which include the criterion of flexibility as well as the intrinsic constraints of micro-objects (i.e roughness, geometry, physicochemical characteristics...). Moreover, it is essential to consider the microscopic forces that interact between the micro objects. These forces also depend on the environment of the micro world (i.e dust, moisture, temperature.). Located between the storage and the assembly system, feeding systems can have a profond effect on the effeciency of an assembly station (Whitney, 2004). The ideal situation is one in which of the part are ready, in the correct orientation, at the moment the ressources need them. No assembly time is wasted (Whitney, 2004). But, rhythm and quantity are dubious variables. Also, a Pick \& Place system of production (like the micro-factory developped inside EUPASS) must be placed in the good 
context of production. This approach is valid only for small and average series with changes of frequent and fast flows. This is the reason why, the micro feeders musn't be dedicated to a particular product but must be as flexible as possible while preserving requested rates. The state of the art carried out allows to emphasize the micro parts feeding systems existing as well as the connected principles with the various functions of feeding. From there and from stated previously constraints, emerges a strategy for the choice of such micro parts feeding systems. This choice is also done according to existing technologies. From explored technologies will be especially exposed the one that were retained.

\section{STATE OF THE ART}

The classification of the whole of micro feeders met is drawn from the thesis of T. Ebefors (Ebefors, 2000). There are two categories according to the contact. The first describes the systems without contact between the micro feeder and the micro product whose acronymis CF (Contact Free). The second category gathers this times the micro feeders indicated by $\mathrm{C}$ (with Contact).

\subsection{Contact Free: CF}

One counts for the processes without contacts with the micro objects three great classes: magnetic (Nakasawa et al., 1999; Kim et al., 1990; Iizuka et al., 1994), electrostatic (Moesner et al., 1996; Moesner and Higuchi, 1997; Gengenbach and Boole, 2000; Desai et al., 1999; Fantoni and Santochi, 2004) and pneumatic levitation (Konishi and Fujita, 1994; Hirata et al., 1998; Fukuta et al., 2003; Chapuis et al., 2003; Sin and Stephanou, 2003).

The magnetic systems allow to translate in one or two directions a mover. This palet is generally a permanent magnet. The different systems elaborated are classified as micro conveyors. The electrostatics systems are based on the effects of electrophoresis and dielectrophoresis. This principles can separete different components. An Ac electric field induces a dipole moment, which in the presence of a field gradient experiences a force towards a hight or low field intensity region (positive/negative dielectrophoresis)(Zheng et al., 2003). The electrostatic approaches are used in the micro and macro world. U. Gengenbach ( Gengenbach and Boole, 2000) realized a palette in levitation by a pneumatic flow and can move it with a electrostatic field. The pneumatic levitation approaches are planar systems of micro conveyances based on the distributed micro motion systems (DMMS). The pneumatic conveyance have some advantages, like: no friction problem, no particle 
generation, generation of force large enough to convey objects (Fukuta et al., 2003).

\subsection{With Contact: C}

These methods are gathered in three main categories: the ciliary motion systems (Ataka et al., 1993b; Benecke and Riethmller, 1989; Böhringer et al., 1994; Suh et al., 1997; Suh et al., 1999; Tabata et al., 2002), the ultrasonic feeders (Haake and Dual, 2003, Kim et al., 2004) and the micro robots (Ebefors, 2000, Ferreira, 2000, Ferreira et al., 2004).

Like the contactless pneumatic DMMS micromotion systems, the ciliary micromotion use arrays of tiny simple actuators that co-operate to move objects over relatively large distances and offers possibility in different directions and orientations. Today, the cilia can be moved by the electrostatic forces, magnetic forces, thermic effect and an original concept: a chemical reaction. The first ultrasonics feeders elaborate by (Böhringer et al., 1998), is a table put in vibration by a piezo. The frequency is as, the ultrasonic waves that are generated are able to break surfacic forces. So the components are insulated by a electrostatic field trap (four electrods placed under the table). The last familly is the micro robots. We can found here walker micro robots, pallets. In one hand, the problem is here the energy supply, but in the other hand the main advantage is their height accuracy.

The different processes we saw are assimilated to conveyances. However some works present innovative concepts like the transfer without contact. The next part describe a new approach in order to design a micro parts feeder. The main difference with these feeders or conveyances is to garantee the maintain function, to feed a lot of familly of product with a good orientation.

\section{A GENERIC APPROACH}

A micro part feeding system must fill two objectives: first, to position the micro objects correctly and in the second place, to orient them. Moreover, it must be able to preserve these two parameters without damage the micro objects. Lastly, if several components have to be treated at the same time, it must be able to insulate them. Entering in the micro feeding system, the objects can be conditionneed, by various manners: bulk, palet or fixed on their support. This is the raison why it can be necessary to order these components before their arrival in the micro part feeding system or inside even of it. After, the feeder separate this components. At the end, the components must be positioned and 
oriented at the right time required by the assembly cell.

It is essential from the very beginning of the design, to build a sufficiently flexible feeding system that isn't dedicated to criteria relating to a particular component or a particular family of components. As previously noted: the selected strategy is influenced by the product. But in the micro world, the negligible physical phenomena from macro scale become dominating (Van der Waals forces, electrostatic forces, capillary forces). From equations of these physical phenomena, the significant characteristics of the micro objects were retained (Regnier et al., 2004, Huang et al., 2004, Zhou and Nelson, 2000):

- Lifshitz-Van Der Waals constant,

- distance between the surface,

- some geometrical characteristics (like the radius of a sphere),

- surface charge density,

- contact angle between a liquid and a micro component,

- surface tension.

Moreover these adhesions forces are also influenced by the environmental conditions (Zhou et al., 2004):

- dust,

- moisture,

- temperature,

- vibrations,

- operation inside air, inert gaz, liquid...,

- pressure,

- permittivity of the environment.

It becomes significant in these scales by including these parameters in the choice of a strategy. The obtained results show that the strategy for the choice of a micro parts feeding system does not only depends on three criteria: weight, size and form. Thus it is important to take into account the plasticity, the elasticity, the surface roughness of the component, the hydrophobic properties of the surfaces and the physicochemical properties of the components. All this parameters influence the way to grip a 
component inside the microworld (Tichem et al., 2004). So they are considered at the beginning of their design. Designing a micro parts feeder, will induce the same problem. Now, it is necessary to find the generic aspects among various technological solutions. A micro feeding system is considered quite generic, according to its independance regarding a maximun of criteria, such conventional (weight, size...) and such micro world criteria (plasticity, hydrophobic surfaces, dust, temperature...). After establishing the correlation between the technological criteria and solutions, it is possible to emphasize the limits of the generic aspect of each solution.

\section{SOME TECHNOLOGIES EXPLORED}

In the framework of the EUPASS project, where objects go several hundreds of microns and few millimeters, the accuracy degree of position is defined according to the size of the objects. This value relates to dimensions of the treated objects. Using the proposed method, according to the constraints of the micro world, and those of european project, various technological solutions were approached. The method is represented by a classification scheme inside which we evaluate the technologies according to the criteria previously quoted. The first row of the baord reopresent the differents technologies and the first columns the differents criteria. This scheme is inspered by (Tichem et al., 2004).

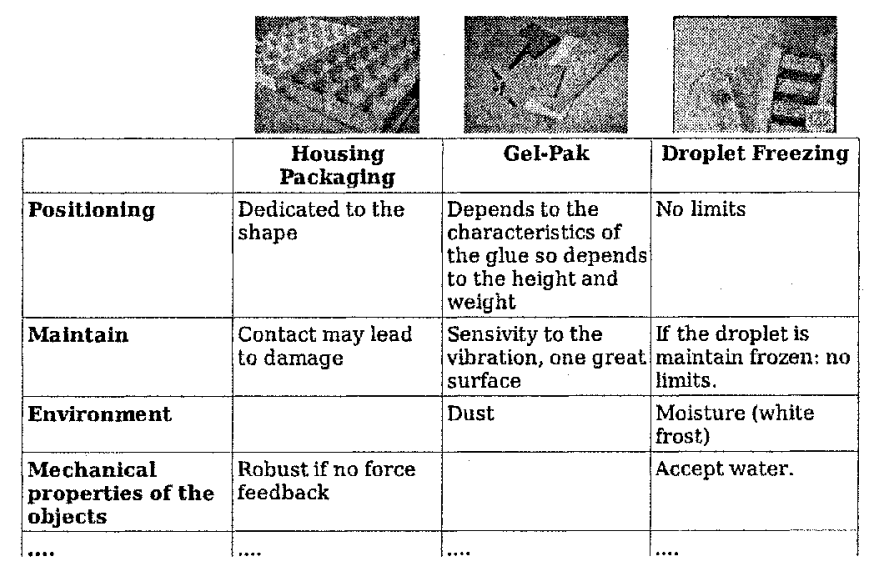

Figure 1. Classification scheme

The figure 1 shows only the most important technologies explored inside the EUPASS project with some criteria. We explored here tree systems 
(housing packaging, Gel-Pak and freezing) and we retain only two; the Gel-Pak and the freezing. The exemples of the analyse show that the housing packaging is not flexible enough and can't be taken into account. Gel-Pak and the frozen droplet offer more possibilities and can cover more different components. Since a feeding system can fulfill several functions, the first one to satisfy is the transport of the components inside an assembly cell. With this intention, the micro components are not treated directly from the assembly cell. It is more preferable to disconnect storage and load of components of assembly cell by using an intermediate support (see Fig. 2). By this way, the filling of this inter-

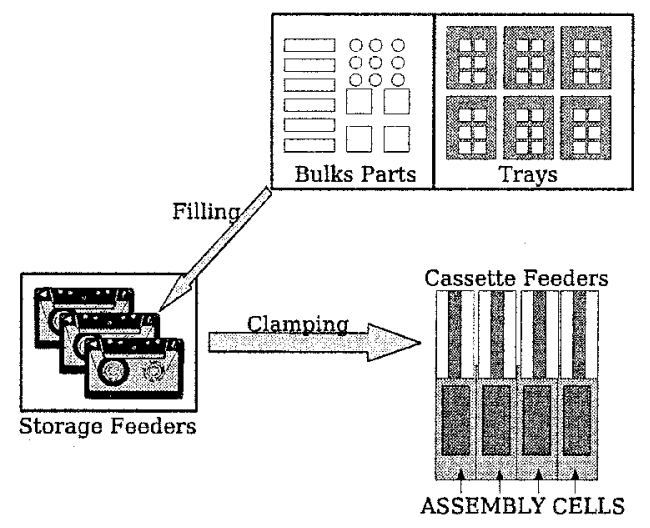

Figure 2. Main frame layout of feeding

mediate support can be adapted in ressources outside and independantly the assembly cell. This is a "Plug \& Produce" solution. The constraints of position, orientation and maintain of objects can thus be guaranteed. Two solutions have been retained. They are sufficiently generic to cover a broad various and varied line of products. They are based on the technology of an adhesive gel (Gel-Pak) and freezing. The final shape of these solutions corresponds to a storage stage, like a video or audio cassette which will be placed thereafter in the assembly cell. A plugin interface realize the positioning. Nicely called "Magic-Carpet", it realises the liaison between stock and cell. These two solutions allows to transport and maintain some differents micro objects. The most ineresting system is the freezing. The Gel-Pak system cannot cover a great familly of components if theirs shapes is between some micrometers to few millimeters. In this case, the adhesive band is dedicated to the height. Moreover, the stability is garanted only if the contact aera is large enough. On the 
contrary, a frozen droplet can maintain any types of micro component without takes into account the shape and the height (more droplets can be used in order to maintain a meso/milli-component).

\section{SYNTHESIS}

Enumerating all the advanges and disavantages of each technologies allows to emerge the principal caracteristics of each. In fact, we characterize a micro parts feeding system according the three criteria of the GFM pyramid: Genericity, Flexibility, Modularity. The first step to design a micro parts feeder correspond to the base of this pyramid: the generic aspect. Then, we go up each stage in order to find the limits of the flexibility and modularity. When the classification scheme is finished, we know the limits of range of the feeders. Finally, this allows to emphasize the degree of flexibility as well as the necessary modularity of each solution. The applied method, used inside the EUPASS project, for the design of a micro part feeding system, could be represented and summarized by this pyramid.

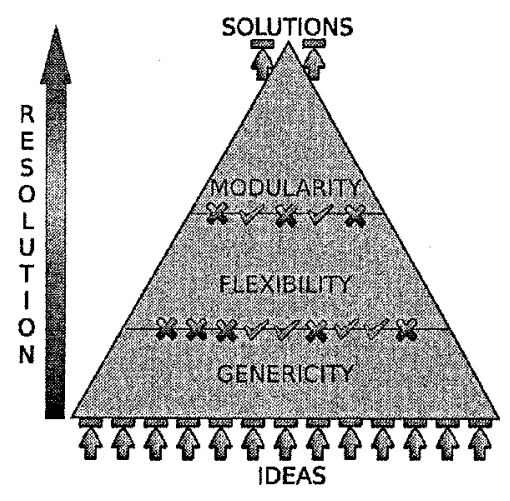

Figure 3. GFM Pyramid

\section{CONCLUSION}

To conclude, this micro feeder is the base of futurs works to develop a module enabling rapid configuration and deployement of flexible precision assembly systems with minimum investment cost. Thus, the "Magic-Carpet" will evolve in order to be integrated in the future cells of micro assembly, with a strong standardization between the various modules. Our approach permits to cover a great number of component 
kinds because it does not necessary realizes a design feedback when the production changes. Several analyses will be necessary in order to test and validate this approach. However, the principles of this solution gives a reusable and generic micro feeder that allows to decrease the design time and costs. It gives too an answer to the most important problem of classical feeders: to be dedicated to one product design.

\section{References}

Ataka, M. et al. (1993a). Fabrication an Operation of Polyimide Bimorph Actuors for a Ciliary Motion System. Journal of Microelectromechanical Systems, 2(4):146150.

Ataka, M., Omodaka, A., and Fujita, H. (1993b). A Biometric Micro Motion System $\sim$ A Ciliary Motion System . In Sensors and Actuators, pages 38-41, Yokohama, Japan.

Benecke, W. and Riethmller, W. (1989). Application of Silicon-Microactuators Based on Bimorph Structures. In Proc. of the IEEE MEMS Workshop, An Investigation of Micro Structures, Sensors, Actuators, Machines and Robots, pages 116-120, Salt Lake City, USA.

Böhringer, K. F., Donald, Bruce R., and MacDonald, N. C. (1996). What Programmable Vertor Fields Can (and Cannot) Do: Force Field Algorithms for MEMS and Vibratory Plate Parts Feeders. In Proc. ICRA, Mineapolis, USA.

Böhringer, K. F. et al. (1994). Sensorless Manipulation Using Massively Parallel Micofabricated Actuator Arrays. In Proc. ICRA, volume 1, pages 826-833, San Diego, USA.

Böhringer, K. F. et al. (1997). Computational Methods for Design and Control of MEMS Micromanipulator Arrays. IEEE Computational Science \& engineering.

Böhringer, K. F. et al. (1998). Parallel microassembly with Electrostatic Force Field. In Proc. ICRA, pages 1204-1211, Leuven, Belgium.

Böhringer, K.F., Fearing, R.S., and Goldberg, K. Y. (1999). Microassembly. In Nof, Shimon, editor, The Handbook of Industrial Robotics, pages 1045-1066. John Wiley $\&$ Sons, seconde edition.

Brussel, H. Van et al. (2000). Assembly of microsystems. Annals of the CIRP, 49(2):451472.

Chapuis, Y-A. et al. (2003). Les Microsystmes Intelligents : Technologies et Applications. In Journées Scientifiques Francophones: JSF.

Desai, A., Lee, S-W., , and Tai, Y-C. (1999). A MEMS ELECTROSTATIC PARTICLE TRANSPORTATION SYSTEM. Sensors and Actuators, A: PHYSICAL.

Donald, B. R. et al. (2003). Power Delivery and Locomotion Unthered Microactuators. Journal of Microelectromechanical Systems, 12(6):947-959.

Ebefors, T. (2000). POLYIMIDE V-GROOVE JOINTS FOR THREE - DIMENSIONAL SILICON TRANSDUCERS. PhD thesis, ROYAL INSTITUTE OF TECHNOLOGY (KTH), Stockholm, Sweden.

Fantoni, G. and Santochi, M. (2004). A contactless linear movement of mini and microparts. In Proc. of the IMGO4, Genova, Italy. 
Ferreira, A. (2000). Design of a Flexible Conveyer Microrobot with Electromagnetic FieldBased Friction Drive Control for Microfactory Stations. Journal of $\mathrm{Mi}^{-}$ cromechatronics, 1(1):49-66.

Ferreira, A., Cassier, C., and Hirai, S. (2004). Automatic Microassembly System Assisted by Vision Servoing and Virtual Reality. IEEE/ASME TRANSACTIONS ON MECHATRONICS, 9(2):321-333.

Fukuta, Y. et al. (2003). Pneumatic Two-Dimesional Conveyance System for autonomous Distributed MEMS. In The 12th International Conference on Solid-State Sensors, Acluors and Microsystems, pages 1019-1022, Boston, USA.

Gengenbach, U. and Boole, J. (2000). electrostatic feeder for contactless transport of miniature and microparts. In Proceeding of SPIE Microrobotics and Microassembly $I I$, volume 4194, pages 75-81.

Goosen, J.F.L and Wlffenbuttel, R.F. (1995). Object Positionning Using a Surface Micromachined Distributed System. In The 8th International Conference on SolidState Sensors and Actwors, and Eurosensors IX, volume 2, pages 396-399, Stockholm, Sweden.

Haake, A. and Dual, J. (2003). Paricle positionning by a two- or three-dimensional ultrasound field excited by surface waves. In $W C U$, Paris, France.

Hill, M. et al. (2003). A microfabricated ultrasonic particle manipulator with frequency selectable nodal planes. In WCU, Paris, France.

Hirata, T. et al. (1998). A Novel Pneumatic Actuator System Realised by MicroelectroDischarge Machining. In Proceeding of the 11th International Workshop on Micro Electro Mechanical Systems (MEMS'98), pages 160-165, Heildelberg, Germany.

Huang, J-T. et al. (2004). Separate and Manipulate Different Kind of Particles by Dielectrophoresis. Tamkang Journal of Science and Engineering, 7(2):87-90.

Iizuka, T. et al. (1994). A Micro X-Y-theta Conveyor by Superconducting Magnetic Levitation. In IEEE Symposium on Emerging Technologies and Factory Automation, ETFA'94, pages 62-67, IIS The University of Tokyo.

Kim, D-H. et al. (2004). High-Troughput Cell Manipulation Using Ultrasound Fields. In Proceedings of the 26th Annual International Conference of the IEEE EMBS, pages 2571-2574, San Francisco, USA.

Kim, Y-K., Katsurai, M., and Fujita, H. (1990). Fabrication and testing of a micro supraconducting actuator using the Meissner effect. In Proceedings of IEEE 3rd International Workshop on Micro Electro Mechanical Sytems (MEMS'90), pages 61-66, Napa Valley, USA.

Konishi, S. and Fujita, H. (1994). A Conveyance System Using Air Flow Based on the Concept of Distributed Micro Motion Systems. Journal of Microelectromechanical Systems, 3(2):54-58.

Liu, C. (1995). A micromachined permalloy magnetic actuor array for micro-robotics assembly systems. In International Conference of Solid-Sate Sensors and Actuors, Stockholm, Sweden.

Masudo, T. and Okada, T. (2001). Ultrasonic radiation - novel principle for microparticle separation. In ANALYTICAL SCIENCES, volume 17 SUPPLEMENT, pages il341-il344. The Japan Society for Analytical Chemistry.

Mitani, A., Sugano, N., and Hirai, S. (2005). Micro-parts feeding by a saw-tooth surface. In Proc. ICRA, Barcelona, Spain. 
Moesner, F, M. and Higuchi, T. (1997). Contactless manipulation of microparts by electric field traps. In Proceedings of the SPIE's International Symposium on Microrobotics and Microsystem Fabrication, volume 3202, pages 168-175, Pittsburgh.

Moesner, F. M., Higuchi, T., and Tanii, Y. (1996). New considerations for traveling wave particle handling. In IEEE Industry Application Society Conference Record, 31st Annual Meeting, pages 1986-1993, San Diego.

Nakasawa, H. et al. (1997). The two-dimensional micro conveyor. In International Conference of Solid-State Sensors and Actuators, Transducer'97, pages 33-36, Chicago, USA.

Nakasawa, H. et al. (1999). Electromagnetic Micro-Parts Conveyer with Coil-Diode Modules. In The 10th International Conference on Solid-State Sensors and Actuators, volume 2 of Transducer'99, pages 1192-1195, Sendai, Japan.

Regnier, S., Rougeot, P., and Chaillet, N. (2004). Modélisation des effets microscopiques pour la micro-manipulation. In Troisième journée du réseau thématique pluridisciplinaire microrobotique.

Sin, J. and Stephanou, H. (2003). A parallel micromanipulation method for microassembly. In Proceeding of SPIE : Micromachining and Microfabrication Process Technology VII, volume 4557, pages 157-164.

Smela, E. and Kallenbach, M. (1999). Electrochemically Driven Polypyrrole Bilayers for Moving and Positionging Bulk Micromachined Silicon Plates. Journal of microelectromechanical systems, $8(4)$.

Suh, J. W. et al. (1997). Organic thermal and ectrostatic ciliary microactuor array for object manipulation. Sensor and Actuors: A, 58:51-60.

Suh, J. W. et al. (1999). CMOS Integrated Ciliary Actuato Array as a GeneralPurpose Micromanipulation Tool for Small Objects. Journal of Microelectromechanical Systems, 8(4):483-496.

Tabata, O. et al. (2002). Ciliary motion actuator using self-oscillating gel. Sensors and Actuators: A 95, pages 234-238.

Tichem, M. et al. (2004). A classification scheme for quantitative analisys of micro-frip principles. Assembly Automation, 24(1):88-93.

Varstenbosch, J-M. et al. (2004). Theory and experiments on vibration feeding off small parts in the presence of adhesive forces. In IPAS 2004, Bad hofgastein, Austria.

Wautelet, M. and Guisbiers, G. (2003). Macro, micro et nanosystèmes: des physiques différentes? SFT: Congrès franais de thermique.

Whitney, D. E. (2004). Mechanical Assemblies. Oxford University Press.

Zheng, L. et al. (2003). Towards Single Molecule Manipulation with Dielectrophoresis using Nanoelectrodes. In Proceedings of the Third IEEE Conference on Nanotechnology, volume 1, pages 437-440.

Zheng, W., Buhlmann, P., and Jacons, H. O. (2004). Sequential shape-and-solderdirected self-assembly of functional microsystems. PNAS, 101(35):12814-12817.

Zhou, Q. et al. (2004). Microassembly system with controlled environment. Journal of Micromechatronics, 2, Issue 3:227-248.

Zhou, Y. and Nelson, B. J. (2000). The Effect of Material properties and Gripping Force on Micrograsping. In ICRA, pages 1115-1120, San Francisco, USA. 